\title{
Les parcours chantés du raid. Expérience, composition et domestication d'un environnement lointain
}

\section{Giordano Marmone}

\section{OpenEdition}

\section{Journals}

Édition électronique

URL : https://journals.openedition.org/clo/8469

DOI : $10.4000 /$ clo.8469

ISSN : 2266-1816

Éditeur

INALCO

\section{Édition imprimée}

Date de publication : 30 juin 2020

Pagination : 123-146

ISBN : 978-2-85831-392-1

ISSN : 0396-891X

\section{Référence électronique}

Giordano Marmone, «Les parcours chantés du raid. Expérience, composition et domestication d'un environnement lointain », Cahiers de littérature orale [En ligne], 87 | 2020, mis en ligne le 25 septembre 2021, consulté le 09 novembre 2022. URL : http://journals.openedition.org/clo/8469 ; DOI : https:// doi.org/10.4000/clo.8469 


\title{
Les parcours chantés du raid. Expérience, composition et domestication d'un environnement lointain
}

\author{
Giordano Marmone \\ Fondation Fyssen - Department of Afroamerican and African Studies, \\ University of Michigan \\ À mon cher ami Saitoti (199? - 2020), \\ un jour parti sur le chemin du raid sans en revenir
}

«Pourquoi vous, les Samburu, appelez-vous les chants des “arbres" ? 》 Pendant longtemps cette question avait continué à s'écraser contre un mur à l'apparence inébranlable d'un « je ne sais pas ». L'habitude des Samburu de faire référence aux chants à travers le terme lcheni (lkeek au pluriel), littéralement « $\operatorname{arbre}^{1} »$, constituait, de mon point de vue, un vrai mystère. J'étais pourtant persuadé que cela ne pouvait pas être dû au hasard. Il devait forcément y avoir une raison, une explication qui m'aurait permis d'éclairer le statut ontologique des compositions musicales chez ce peuple de pasteurs transhumants du Kenya septentrional.

En 2013, je trouvai enfin le courage d'organiser un entretien avec Huran Lepile (192? - 2014), un ancien forgeur de lances et d'épées, l'homme le plus âgé du mont Nyiro, au nord du Samburu County. Les appartenants à son

1. Le terme lcheni/lkeek se traduit principalement par « arbre » et, par extension, par « plante », en particulier en référence aux plantes médicinales (Payne \& Ole-Kotikash, 2008). Dans son association terminologique aux chants, les Samburu du mont Nyiro, auprès desquels je conduis mes terrains, identifient le mot lcheni/lkeek avec les arbres. 
groupe générationnel, appelé lmekuri ${ }^{2}$, étaient désormais presque tous morts et Lepile, en tant que dernier membre de cette promotion dans son village, jouissait du plus grand respect. Quand je lui posai ma question sur les chants-arbres je ne compris pas complètement sa réponse. À cette époque ma maîtrise du samburu était encore très faible et je n'en fus pas trop surpris. Le vrai problème demeurait ailleurs. Pour m'expliquer son point de vue à ce propos, Lepile fit recours à un langage figuré tellement peu usuel que j'eus besoin de l'aide de deux traducteurs pour comprendre exactement le sens de ses phrases :

Ceci [c'est] un arbre parce que [c'est] une chose qui est là quand on germe. Quand [les gens] germent, le chant ${ }^{3}$ [aussi] est là. Donc en les trouvant tous les deux, [arbres et chants] deviennent une seule chose ${ }^{4}$.

Malgré leur caractère légèrement cryptique, ces paroles ouvrèrent pour moi une fenêtre sur la complexité des catégories locales associées au statut et au rôle des chants et me fournirent une lecture inédite de la place qu'ils occupent dans le processus de façonnement des individualités et du système social des Samburu. On y reviendra d'ici peu.

La production musicale des populations « à classes d'âges » d'Afrique de l'Est (et notamment des communautés de langue maa comme les Maasai et les Samburu) a été souvent décrite comme un prolongement narratif de la pratique du raid de bétail, qui encore de nos jours constitue un passage essentiel dans la construction de la masculinité au sein des communautés pastorales et agro-pastorales d'Afrique orientale (Spencer, 1965, 1985 ; Wasamba, 2009 ; Peatrik, 2013). Quand j'ai entamé mon étude des textes chantés générationnels des Samburu, je m'attendais donc à me confronter aux récits de grands exploits militaires, aux descriptions de batailles menées jusqu'au dernier souffle à coups de lances et de fusils automatiques, aux éloges des actes de bravoure des « guerriers ». En réalité le thème du conflit, bien qu'omniprésent, est traité dans les « chants de raid » selon des modalités fort différentes de ce à quoi l'on pourrait s'attendre.

2. Cette promotion générationnelle a été instaurée en 1936 et a été ouverte au recrutement pendant environ les dix années suivantes.

3. Ici Lepile, vraisemblablement pour une question de clarté, utilise le mot sungolio, le terme canonique pour définir les chants.

4. Lcheni na ele amu ntoki notopukunyieki eatai, naa kotupukunyieki sungolio eatai, naa kotumo pokorare eaku tonobuo. Entretien enregistré, 27/07/2013, min 16.28-16.38. 
Les études consacrées à l'analyse des chants des peuples pastoraux d'Afrique de l'Est (pas très nombreuses à vrai dire) ont, sans exception, souligné le lien d'interdépendance qui existe entre conflit, vie pastorale et création poético-musicale 5 . Je n'ai pas l'intention de mettre en discussion ce point. La pratique du vol de bétail représente effectivement une des sources les plus importantes d'inspiration pour la création des chants masculins dans la terre des Samburu et ailleurs. Cependant, dans les faits, la littérature scientifique ne nous dit presque rien sur la façon dont l'expérience du raid est développée à travers le chant. Comment les razzias et leurs enjeux socio-politiques sont-elles verbalisées musicalement par les Samburu ? Comme nous le verrons, il ne s'agit pas d'une question anodine. Mon objectif dans cet article est de montrer que les chants générationnels inspirés par l'expérience du conflit ne sont pas de simples descriptions d'événements à caractère militaire. Ils relèvent plutôt d'un dispositif d'analyse du réel qui s'appuie sur l'exploration et sur la perception de l'environnement pour fournir aux membres de la communauté les instruments cognitifs et culturels pour interpréter le présent. L'analyse systématique des textes de ces chants, qui a constitué une partie essentielle de mon travail de recherche au cours des dix dernières années, m’a permis en effet de constater que, dans les « chants de raid », le raid lui-même n'est quasiment jamais évoqué de manière explicite. Les solistes, en revanche, parlent longuement de plantes et de fleuves, de montagnes et de vallées. Les ennemis occupent une place marginale. Les combats sont rarement mentionnés. Le bétail volé n'est cité qu'à travers des allusions. La narration d'une razzia se présente, dans les faits, à travers des références à l'environnement et ses composantes ainsi qu'aux sujets politiques impliqués (les membres du statut des Pères, les groupes de jeunes circoncis, les représentants du gouvernement national et régional, l'armée kenyane, etc.). Ces textes chantés, associés aux mouvements dansés qui accompagnent leur exécution, établissent une relation avec les territoires décrits qui n'est pas seulement analytique mais aussi corporelle et sensorielle. Les sauts mais surtout les longues marches rythmées qui composent une large partie des schémas chorégraphiques des Samburu, contribuent en effet, selon l'hypothèse que je vais proposer ici, à transformer l'évocation poétique des environnements traversés par les jeunes hommes circoncis en une expérience sensible partagée au sein de la communauté.

Qu'est-ce que donc un raid pour les peuples pastoraux d'Afrique orientale ? Et de quelle manière la parole chantée et la danse font de la perception de

5. Voir aussi Gourlay, 1999 et ECZET, 2019 à propos de l'importance des bovins dans l'activité compositionnelle des populations pastorales d'Afrique orientale. 
l'environnement et du monde végétal un élément essentiel dans le processus de reproduction du système social des Samburu?

\section{Des chants-arbres qui chantent arbres, montagnes et fusils}

Revenons aux phrases prononcées par Huran Lepile. Sa réflexion procède à un examen de l'être humain dans son rapport avec le monde et la musique : les chants sont comme les arbres car ils constituent tous ensemble des éléments de l'environnement social et naturel des individus. Les hommes et les femmes ne naissent pas, ils «germent » [kotupuku] - eux aussi comme des entités végétales et prennent place dans un macrocosme au sein duquel les frontières entre artefacts propres à l'action humaine et éléments du monde naturel s'évanouissent. Les chants, dans le vocabulaire samburu, deviennent « comme les arbres » ou plutôt, ils sont des arbres (au point que, pour exhorter quelqu'un à chanter, on dit parfois tosho lcheni !, « frappe l'arbre ! »). Ils se conçoivent ainsi presque comme des composantes organiques du territoire des Samburu. L'existence des chants-arbres précède, selon Lepile, la naissance des êtres humains car les chants ont toujours été présents dans la vie sociale des Samburu, tout comme les arbres ont toujours fait partie de leur environnement. Ce qui constitue la substance conceptuelle de cette corrélation ontologique doit donc être recherché dans la faculté des arbres et des chants à apparaître comme des entités qui relient les individus et les générations avec le passé mais en même temps existent dans le présent et se développent vers le futur. Dans une société organisée en classes d'âges, ce plan temporel intergénérationnel modélisé par les chants-arbres constitue le fondement des catégories politiques, statutaires et cérémonielles essentielles pour son fonctionnement. Au même titre que les arbres et les individus, les répertoires générationnels des Samburu se développent, se ramifient en d'innombrables formes et variations, vieillissent et meurent en laissant place à de nouveaux chants - ou si l'on veut à de nouvelles « espèces » musicales - qui iront repeupler leur contrée.

Au moment de leur naissance, les membres de cette communauté sont insérés dans un milieu biologico-musical en mutation constante qu'ils contribueront à façonner au fil du temps, selon leurs nécessités et leurs goûts, avec les membres de leur promotion générationnelle, une fois qu'ils en auront les capacités et les compétences. Les savoirs liés aux soins du bétail, aux plantes et au territoire développés à travers la composition de nouveaux chants font l'objet d'un parcours d'appropriation, d'apprentissage mais aussi d'adaptation au contemporain assimilé et incorporé (Ingold, 2000) par le chant et la danse. 
Les textes chantés créés par les hommes pendant leur état de jeunes circoncis ${ }^{6}$ se caractérisent par des références systématiques aux éléments de l'environnement avec lesquels ceux-ci ont interagi ou qu'ils ont simplement observés pendant leurs périples dans les savanes et les forêts du Kenya du nord, en quête des villages et du bétail de leurs ennemis. La dimension du voyage et de l'analyse du territoire est prépondérante dans la production poético-musicale des Samburu. Pourtant la présence de références constantes aux composantes de l'environnement ne fait pas de ces chants des « cartes musicales ». Le récit chanté d'une razzia de bétail n'a pas comme objectif la reconstruction ponctuelle d'un itinéraire décrit dans ses détails, permettant à l'auditoire d'imaginer un parcours précis et reproductible. À travers leurs paroles et l'évocation des composantes du territoire qu'ils ont traversé, les jeunes circoncis veulent montrer qu'ils ont « dit la vérité $\gg$. Mais comment ? La réponse est plus complexe que ce que l'on pourrait croire. Il est donc opportun de commencer à analyser quelques exemples de texte générationnel. Ceux que je propose ici ont été composés par les membres de la promotion lkishami, qui ont été jeunes circoncis de 2005 à 2019 et sont à présent passés au statut d'âge des Pères. La création des textes générationnels a lieu presque exclusivement pendant la période de l'état de jeunes circoncis, qui peut durer de sept à quinze ans. La grande partie de l'activité compositionnelle qui donne forme au répertoire d'une promotion au niveau local se concentre dans cette phase du parcours d'ontogénèse des hommes. Après leur passage à l'état de Pères elle s'arrête presque complétement. Cette transition produit aussi une diminution drastique des occasions de déplacement. À l'issue de ce changement de statut, les hommes ne partent plus en raid et ne suivent pas les transhumances du bétail comme pendant les années du lamurrano, l'état de jeunes circoncis. À une réduction de la mobilité et des possibilités d'entrer en contact avec des territoires lointains avec les membres de sa propre promotion, correspond une baisse de l'activité créative.

6. À travers la circoncision (entre 14 et 20 ans environ) les hommes intègrent une promotion générationnelle et entrent dans l'état de jeune circoncis (Imurrani). Sept/ quinze ans après (selon le moment de leur initiation) ils accèdent avec tous les membres de leur promotion à l'état de Pères. 


\section{Lkulosi :}

Je ne laisse pas ceux qui contrôlent ${ }^{7}$

Les lmurran $^{8}$ [dans la] terre au sol rouge et aux pierres noires près du torrent sec

[Dans la] terre au sol rouge et aux pierres noires, [dans le] territoire plat [aux plantes] lkino $i^{9}$

Pour que je puisse trouver et surveiller [les vaches des ennemis ${ }^{10}$ ]

Nous nous sommes installés sans permission

[Å] Ntepes sans bénédiction [des Pères] ${ }^{11}$

Nous avons escaladé sans permission

Près des torrents secs [et des] collines

À Namalit [au] crépuscule

La vache-aux-cornes-qui-pointent-vers-le-bas du [frère] de Njilena

Si le groupe de raiders revient en arrière

Le tort ne les tuera pas ${ }^{12}$

S'ils craignent [la terre où les ennemis] habitent
Mapal masopirie

Marti e lkijat lmurran

Marti e lkinoi a ata

nkop

Paye atum namarrarrie

Kitamanye meseli

Ntepes namayana

Kitidire meseli

To lkijata ltaleta

Ta Namalit emutie

Nkarroia e lo Njilena

Tenetorrinye lwampa

Nemetari mokosa

Teneturia namanya

7. Les jeunes circoncis envoyés espionner les ennemis pour compter leurs têtes de bétail et suivre leurs déplacements.

8. Les membres du statut d'âge des jeunes circoncis. Ce terme dérive de muratare, circoncire, et de lmurra, le pénis des hommes circoncis.

\section{Lannea alata, Payne \& Ole-Kotikash, 2008.}

10. Dans ce cas le soliste fait référence à un raid contre les Turkana, une communauté d'agro pasteurs qui habitent dans la région éponyme à l'ouest des terres des Samburu. Le nom de ce peuple n'est pourtant jamais évoqué.

11. L'activité militaire des Samburu s'appuie de nos jours sur quatre types différents de raid : les raids effectués avec la bénédiction et sous mandat des Pères; les raids interdits par les Pères et menés de manière secrète par les jeunes circoncis; les grandes campagnes de vengeance qui voient la participation de tous les hommes adultes sans distinction de promotion ou de statut d'âge; les nkutuyat, les missions de récupération du bétail volé aux membres de la communauté par les ennemis.

12. Nous ne pourrons les blâmer. 
LES PARCOURS CHANTÉS DU RAID. EXPÉRIENCE, COMPOSITION ET DOMESTICATION D'UN ENVIRONNEMENT LOINTAIN

Giordano MARMONE

Ils se préparent à Nasai

Quand j'ai trouvé [la terre où les ennemis] habitent

J'ai ramené la joie [aux] lmurran

[Le fusil] vert de Lepatoye

A sauvé les lmurran

Nous avons escaladé [avec le fusil] aux trous ${ }^{13}$

Dans les basses-terres [aux] collines [...

\section{Lkukorr onyekie:}

[...]

Écoute comment j'ai commis le vol

Nous avons traversé trois eaux [fleuves] à

Ntipadas

J'ai escaladé [le mont] Mekedi et j'ai trouvé ce que je voulais

Nous avons transpercé [les ennemis là où se trouvent les arbres] lmasanduku ${ }^{14}$ derrière Nanyuki

J'ai transpercé [les ennemis à] Karashira [aux] lpopoyi $i^{15}$ secs

Nous avons tiré [avec les fusils] à Ratia après l'arrivée du jour

J'ai tiré des marques de feu [des balles] pour [les vaches] de Mugie
Ta Nasai etanape

Tanatumo namanya

Naeakie jida lmurran

Nanyore e Lepatoye

Naitoipoye Imurran

Kitidirie nairimuo

To lpurkelli ltaleta

[...]

[...]

Toniyo naikunaie

patipatisho

Kitalaya nkariak uni te Ntipadas

Mekedi atidira nasipu nayeu

Kiteremo lmasanduku loitagu Nanyuki

Naremie Karashira

Ipopoyi otoio

Kutoyoro Raitia nushi naikiniunye

Nayorie ne Mugie nampay e nkuma

13. Le soliste fait ici référence au fusil automatique du type $G 3$.

14. Podocarpus falcatus, NANYINGi et al., 2008.

15. Euphorbia candelabra, Payne \& Ole-Kotikash, 2008. 
[Que] la personne qui n'aime pas que je tire le feu [avec le fusil]

Aille [chercher] le constructeur qui se trouve en Éthiopie ${ }^{16}$
Ltujani oibaie

nayorishere nkuma

Tapaki lpundi loweni te Ethiopia

Les textes transcrits ci-dessus relèvent de deux chants (lkulosi et lkukorr onyekie) qui ont été exécutés respectivement en 2013 et en 2012 lors d'une session de danse-chant informelle des lmurran et des filles célibataires, dans les hautes-terres du nord de la région samburu (dans le premier cas) et à l'occasion d'une cérémonie de circoncision, dans les basses-terres du sud (dans le deuxième). Ces deux chants prévoient la présence d'un chœur et de plusieurs solistes qui alternent. Lkulosi s'articule, dans la plupart des cas, autour d'une série de distiques heptasyllabiques exécutés à tour de rôle par deux solistes. Les phrases plus courtes, comme par exemple Ntepes namayana, formée de six syllabes, atteignent la longueur exigée par la structure métrique et mélodique du chant par l'ajout de syllabes non signifiantes. Lkukorr (ici dans sa section onyekie [rouge]) se caractérise par un contour mélodique plus proche de la déclamation, à mi-chemin entre le parlé et le chanté. D’un point de vue métrique, donc, ses phrases sont plutôt variables.

Ces deux textes nous donnent un aperçu assez complet des différentes façons selon lesquelles les solistes samburu peuvent créer le récit d'une razzia et la place qu'ils accordent aux composantes de l'environnement, aux toponymes et aux détails de type militaire. Les deux jeunes circoncis qui exécutent lkulosi évoquent le raid auquel ils ont participé de manière indirecte, sans jamais mentionner ni le nom de la communauté attaquée ni le lieu exact du vol de bétail. Ses aspects tactiques et son déroulement n’émergent qu'à travers des allusions («Quand j'ai trouvé [la terre où les ennemis] habitent/J'ai ramené la joie [aux] lmurran ») ou des fragments narratifs (le recours aux scouts, la surveillance à distance des ennemis, les hésitations du groupe des acteurs du raid, un des fusils utilisés, etc.). Les événements décrits restent pourtant parfaitement reconnaissables par l'auditoire grâce à l'énonciation des noms des lieux que les lmurran ont traversés et du type de végétation (les plantes lkinö̈, de l'espèce lannea alata, de la famille des Anacardiacées) qui caractérisait ces territoires. Le deuxième texte évoque le raid de façon beaucoup plus explicite. Ses phrases constituent donc un bon exemple de la variabilité stylistique qui peut caractériser ces compositions. Même dans ce cas nous ne connaissons pas le nom des victimes de la razzia mais, contrairement au chant précédent, le soliste tient à nous faire savoir dans quel

16. Aille chercher celui qui a fabriqué le fusil en Éthiopie. 
lieu il les a transpercés avec sa lance. Dans deux cas c'est une espèce végétale (en l'occurrence le podocarpus falcatus, un conifère de la famille des Podocarpacées, et l'euphorbia candelabra, une plante succulente de la famille des Euphorbiacées) qui constitue l'ancrage spatial de ses actes. Le soliste conclut son intervention avec une fine analyse du contexte géopolitique à l'origine - du moins d'après ses connaissances - de la diffusion des armes à feu dans son territoire. Cette réflexion sur les changements qui intéressent la pratique contemporaine du conflit se développe encore une fois à travers l'évocation d'un lieu lointain (l'Éthiopie).

Il est évident que le style plus ou moins allusif à travers lequel les jeunes circoncis construisent le récit de leurs combats dans les chants générationnels n'est pas entièrement imputable à une exigence de discrétion. Il s'oppose en effet à une représentation assez fidèle des caractéristiques du territoire (montagnes, collines, fleuves), de la végétation et du type de bétail volé ainsi qu'à la mention constante des toponymes. Tout cela rend l'identification de l'événement décrit plutôt aisée. Les priorités narratives des lmurran ne sont donc pas déterminées par la nécessité de dissimuler leurs actes de violence. Les « chants de raid » ne contiennent pas le récit d'un conflit mais celui d'un parcours dans un territoire (en termes spatiaux mais aussi socio-politiques) peu connu ou considéré comme dangereux qui, à travers les paroles des solistes, peut être pensé et domestiqué (au sens étymologique du terme ${ }^{17}$ ). Pour une communauté pastorale semi-nomade qui, jusqu'à des temps très récents, faisait du déplacement un des piliers de son système socio-économique, le pouvoir de « rapprocher » des terres distantes à travers la traduction en chants des expériences des jeunes circoncis permet d'exercer une domination (même si seulement potentielle) sur des possibles lieux de vie. La pratique poético-musicale et compositionnelle devient ainsi une modalité de contrôle territorial, un contrôle qui doit être cognitif avant de devenir réalisable. L'action militaire n'est que l'arrière-plan narratif, le cadre à l'intérieur duquel la flore, les fleuves et les collines occupent le rôle de protagonistes à côté des lmurran eux-mêmes, leurs armes et les entités socio-politiques avec lesquelles ils interagissent.

Le statut du raid en tant que dispositif d'analyse des espaces et de la société émerge aussi en dehors du contexte musical. Le thème du conflit, et notamment du vol de bétail, est évoqué souvent aussi dans les assemblées politiques des Pères et des jeunes circoncis. Ces occasions formelles permettent à la communauté de construire un récit (et donc une réflexion) sur la contemporanéité exactement comme le fait la composition, l'exécution et l'écoute des chants générationnels.

17. Rendre familier, rendre propre, appartenant au foyer familial, du latin domus, domicile, demeure. 
Il n'est donc pas surprenant de découvrir que les orateurs d'assemblée les plus chevronnés et les solistes-compositeurs les plus doués soient souvent les mêmes personnes, des individus qui, à travers leur maîtrise de la parole, parviennent à acquérir une autorité politique qu'ils exercent par le langage parlé et par le chant (Marmone, 2020). En ayant recours aux mêmes modalités de construction du discours, les orateurs-solistes relient l'expérience du conflit à un examen du territoire qui permet d'ancrer la perception des événements racontés aux grilles gnoséologiques locales. À titre d'exemple je propose ici un extrait de l'intervention d'un soliste et ancien représentant politique des jeunes circoncis (aujourd'hui entré avec sa promotion dans l'état des Pères) lors du Peace \& Reconciliation Meeting, organisé par les autorités du Samburu County dans un village du mont Nyiro pour mettre un frein aux affrontements entre Samburu et Turkana.

[...] Nous avons vécu longtemps avec les Turkana, mais ils ont commencé à nous amener la merde [le conflit]. Ces Turkana de Marti Lorroki viennent ici et partent sans problèmes. Quand les Turkana viennent ici et se déplacent derrière Kawap, alors que la poussière est encore dans l'air ils viennent voler ici. S'ils passent par ici pour aller à Moïtié, ils nous volent [le bétail] et vont à Moïtié. S'ils viennent de Moïtié pour aller à Marti Lorroki pour s'occuper de leurs vaches, ils nous volent et vont à Baragoï. [...] Nos vaches sont passées par Marti Nanyekie, sont entrées [dans la vallée] Suguta et l'ont traversée. Les personnes [les membres de la communauté samburu] les ont suivies, sont arrivées là-bas mais elles n'ont pas pu récupérer les vaches. Ces personnes [les Turkana qui disent vouloir collaborer avec les Samburu] connaissent notre terre. Aucune d'entre elles n'est venue ici pour nous ramener ces gens [les raiders], même pas une. Les Turkana savent-ils qui a pris nos vaches? Ils nous disent « nous avons découvert qui a pris vos vaches » mais nous ne les avons plus vues [...]. (10/07/2014)

L'orateur (qui ne montre aucune intention d'intégrer l'esprit de paix et de réconciliation de la réunion) expose dans ce cas la reconstitution synthétique d'une série de raids subis - et non pas effectués - par sa communauté au cours des mois qui ont précédé l'assemblée. Cependant la mise en mots de ces événements, loin de se présenter comme un simple constat des conditions d'insécurité vécues par les Samburu, s'appuie toujours sur des références spatiales qui, comme dans le cas des chants, traduit un processus d'analyse du territoire. Il y a certainement des différences importantes entre la définition de l'espace qu'on retrouve dans 
les textes chantés et celle qui émerge de cet extrait. Le langage utilisé lors de l'assemblée du Peace \& Reconciliation Meeting est beaucoup plus explicite, donne des indications claires sur les assaillants et leurs déplacements et ne fait aucune référence au monde végétal. Les lieux mentionnés ne sont pas identifiés par le type de végétation qui les caractérise ni par les fleuves qui les traversent. Il s'agit, en effet, de territoires « domestiques », dans lesquels les Samburu habitent depuis longtemps et qu'ils connaissent sur le bout des doigts. Ils ne nécessitent donc pas d'être connotés au-delà de leur nom. La référence aux toponymes a toujours constitué une composante fondamentale du discours politique. En particulier en époque coloniale, quand la connaissance des noms vernaculaires des lieux devint un outil de négociation crucial à travers lequel les Samburu corroboraient leurs revendications territoriales face aux tentatives d'expropriation menées par les représentants du gouvernement anglais (Straight et al., 2016). Les références aux plantes, au collines et aux fleuves semblent, par contre, être réservées aux descriptions des territoires en dehors des espaces de vie habituels. Les composantes de l'environnement représentent donc, dans les chants de raid, une sorte d'ancrage (Basso, 1988) pour relier des événements et des lieux extra-ordinaires à des éléments ordinaires.

Que signifie donc «dire la vérité » du point de vue des jeunes circoncis samburu ? Chaque génération a le devoir de développer des formes de savoir nouvelles. Comme me l'expliquait l'exécuteur d'un des deux chants transcrits plus haut, « on ne peut pas être des solistes si on reste assis ! ». La création et l'innovation, doivent forcément être le produit d'un voyage, d'un parcours et d'une réflexion en dehors des frontières de la quotidienneté. Ces éléments de nouveauté - tels que les changements dans la flore et la faune d'un certain territoire, les déplacements d'une population voisine et de leur bétail, la découverte de nouveaux pâturages et chemins, l'assèchement des fleuves ou des points d'eau, l'usage des produits de la technologie occidentale et asiatique comme les fusils automatiques et les téléphones portables, etc. - sont introduits à travers l'évocation de ce qui est connu (des espèces de plantes par exemple) et qui fait déjà partie de l'expérience des membres de la communauté. «Dire la vérité », en somme, correspond dans ce cas à faire référence à un corpus de savoirs partagés (en particulier liés à l'environnement) qui rendent crédibles et « familiers » les événements, les objets, et les actes décrits par le chant.

Une chose est certaine : mis à part les échanges informels, dans le chant tout comme dans les assemblées politiques (deux loci de formalisation du discours) le récit d'un raid engendre toujours un examen des espaces et de leurs composantes qui est aussi, d'un certain point de vue, la narration d'une société et de ses aspirations. Ces discours, qui se déploient par le chant et par le langage parlé, 
acquièrent leur valeur gnoséologique et leur statut formel à travers l'action des solistes, qui sont souvent aussi des figures de référence tant au niveau musical que politico-institutionnel. Ils sont ceux qui prennent en charge le récit de la « vérité ». Leur voix évoque leur personne (même si parfois non repérable au sein d'un chœur), leur personne mobilise leur statut, leur statut institutionnalise la narration de l'environnement et cette dernière contribue à « domestiquer » l'expérience et le vécu qui, dans ces espaces, ont eu lieu.

\section{Générations et environnements}

Le système des classes d'âges qui caractérise la société des Samburu produit une hiérarchisation des hommes sur la base de leur statut d'âge et de leur appartenance à une promotion générationnelle déterminée. Tous les quatorze ans en moyenne une nouvelle promotion est instaurée et ses membres, affiliés après avoir été circoncis, gravissent ensemble les échelons qui composent le parcours de maturation masculin, de l'état de non-circoncis (qui précède le recrutement dans un groupe générationnel) à celui de jeunes circoncis, lmurran, puis de Pères, Ipayani. À chaque changement d'état de la promotion cadette, toutes les autres progressent d'un échelon. Ce processus d'avancement d'un statut d'âge à l'autre va de pair, chez les hommes, avec un éloignement progressif du foyer familial et du village, puis un retour en arrière (lui aussi graduel) une fois atteint l'état des Pères.

L'état de lmurrani est étroitement lié à la brousse ${ }^{18}$ et à l'exploration des territoires lointains ${ }^{19}$. Les raids, la recherche de nouveaux pâturages pour le bétail, les transhumances saisonnières sont autant d'expériences qui amènent ces jeunes hommes à se confronter avec les différentes formes d'altérité qui proviennent de l'extérieur. Plus qu'une avant-garde militaire, les jeunes circoncis apparaissent donc comme une avant-garde culturelle qui, en concours avec

18. Chez les Meru du Kenya central, les membres de ce statut d'âge étaient appelés nthaka (sg. muthaka), qui veut dire littéralement « broussards », PEATRIK, 1999.

19. Cela est illustré aussi par le fait que les jeunes circoncis scolarisés (qui ne partent pas en raid et ne s'occupent pas du bétail) s'engagent parfois dans de longs voyages à pieds vers des villes distantes de centaines de kilomètres et qui pourraient être facilement atteintes en quelques heures en voiture ou par les transports en commun. Le choix d'affronter ces déplacements difficiles et dangereux (qui les amènent à traverser des terres dont ils ont seulement entendu parler, à interagir avec des personnes inconnues, à dormir à la belle étoile et à devoir chercher chaque jour de quoi se nourrir) exclusivement à la force de leurs jambes est justifié, de manière assez explicite, par le désir de visiter de nouveaux endroits et d'en parler à leur retour. 
les filles célibataires, protagonistes elles aussi du processus de renouvellement des répertoires poético-musicaux et ornementaux générationnels, fournit à la communauté les instruments pour interpréter le présent à travers son intense activité créative et leur relation avec ce qui existe au-delà des itinéraires habituels de leur communauté.

Cette relation expérientielle et analytique avec l'environnement est renouvelée de génération en génération. Les Samburu disent souvent que « chaque promotion combat sa guerre », en faisant référence aux ennemis (les Turkana, les Borana, les Somalis, les Dassanetch, etc.) auxquels, au fil des siècles, les différents groupes générationnels de cette communauté ont dû faire face et aux modalités selon lesquelles ces conflits ont été menés. Ces différentes expériences en matière d'activité militaire ont contribué à produire des formes de savoirs générationnels liées à l'environnement. Comme l'écrit Terrence McCabe : Raiding not only results in the loss of human lives and livelihoods but also exerts a very strong influence on how the land is and is not used (2004, p. 91). Des terres destinées au pâturage peuvent être abandonnées pendant des années, une fois devenues inaccessibles à la suite de longues saisons de conflit et des nouvelles contrées peuvent commencer à être explorées et exploitées pour la subsistance du bétail. La pratique prolongée du raid a donc des conséquences directes sur l'environnement : le cours de fleuves peut être modifié, des points d'eau artificiels peuvent être construits ou détruits, des portions de forêt peuvent être défrichées ou incendiées, des types de bois peuvent être surexploités pour la construction des huttes ou pour l'allumage du feu, etc. L'inverse est aussi vrai. Les changements des écosystèmes dus aux fluctuations du climat est-africain ont parfois été la cause d'altérations dans la pratique du conflit et de l'exacerbation d'interactions belliqueuses entre populations pastorales, phénomène que certains chercheurs ont qualifié du terme «écoviolence » (Opiyo et al., 2012). Chaque promotion, donc, « combat sa guerre » et explore un environnement différent, même quand les territoires que ses membres traversent ont été déjà sillonnés par les promotions précédentes. Dans la société samburu, qui a toujours réservé à l'économie agricole une position extrêmement marginale, les références chantées au monde végétal se présentent comme une forme alternative d'assujettissement de l'espace qui doit être réaffirmée de génération en génération. Le succès des systèmes économiques fondés sur l'élevage de bovins, dromadaires et petits ruminants en Afrique orientale est la conséquence directe d'un environnement changeant et en déséquilibre (McCabe, 2004), où des périodes d'abondance alternent avec de longues années de sècheresse et de faible pluviosité. La mobilité est donc une stratégie de survie essentielle dans ces régions au climat imprévisible. Pourtant elle ne se réduit pas, comme nous l'avons vu, au nomadisme pastoral en tant que tel, mais implique une relation complexe avec l'espace, fondée sur une 
œuvre d'élargissement constant des frontières du pensable et du domestique, qui trouve sa source dans les déplacements des jeunes circoncis vers des lieux lointains et dans la traduction de ces expériences en textes chantés.

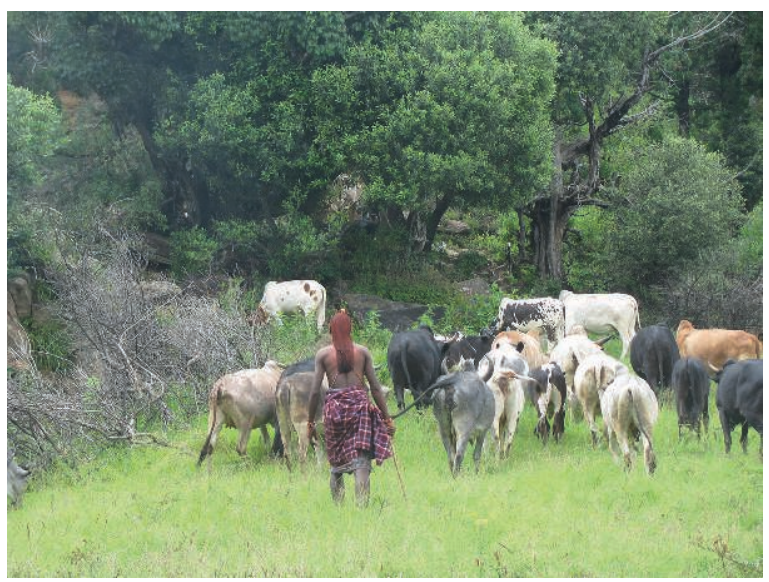

Figure 1

Un jeune circoncis se déplace avec son bétail pour le conduire au pâturage

Photographie : Giordano Marmone (Mont Nyiro, Samburu County septentrional, juillet 2012)

À partir d'un angle de vue similaire, Tim Ingold écrit dans son ouvrage célèbre The Perception of the Environement:

$[T]$ he differences between the activities of hunting and gathering, on the one hand, and singing, storytelling and the narration of myth on the other, cannot be accommodated within the terms of a dichotomy between the material and the mental, between ecological interactions in nature and cultural constructions of nature. On the contrary, both sets of activities are, in the first place, ways of dwelling. The latter, as I have shown, amount not to a metaphorical representation of the world, but to a form of poetic involvement. But it is no different with the activities of hunting and gathering, which entail the same attentive engagement with the environment, and the same exploratory quest for knowledge. In hunting and gathering, as in singing and story-telling, the world "opens out" to people. Hunter-gatherers, in their practices, do not seek to transform the world; they seek revelation. (2000, p. 57)

Dans le cas pris en considération par l'anthropologue anglais, la création de chants qui accompagne la relation des chasseurs-cueilleurs avec l'environnement ne correspond pas à une « construction culturelle de la nature » mais à une 
manière de « demeurer » dans l'espace, qui procède d'une « implication poétique » avec les milieux de vie. La composition de textes chantés ne relève donc pas exclusivement de l'ordre de la représentation mais joue un rôle dans un plus large processus d'incorporation de l'environnement et de découverte (Ingold, 2000, p. 56/193). L'exemple des Samburu montre que la création poético-musicale ne se réduit pas à une modalité de dwelling en tant que telle. La relation mobile avec l'espace et son élaboration musicale permettent également de fabriquer des projections vers des futurs potentiels, de mettre à l'épreuve des possibilités de « demeurer » non seulement dans ce qui « est », mais aussi dans ce qui « pourrait être » ou « sera ». Cela représente une condition sine qua non pour des communautés pastorales qui fondent leur survie sur le nomadisme (mais on pourrait dire la même chose des chasseurs-cueilleurs) dans des écosystèmes en déséquilibre, exposés à des altérations constantes. Comme le montrent Straight et al., l'environnement des Samburu, avec la mobilité qu'il impose, est un producteur de rencontres intercommunautaires, de conflits et de nouvelles identités « ethniques ». Cette agentivité des espaces engendre une analyse du passé et du présent et, en même temps, oblige à lever le regard sur les défis à venir (Straight et al., 2016).

Les promotions générationnelles, mis à part leur fonction primaire de classer les hommes sur la base de leur âge, se présentent comme des formations sociales dont la fonction est d'entraîner une production et une mise à jour des connaissances locales sur l'environnement, la technologie, le bétail et les équilibres politico-militaires à l'échelle régionale et nationale. Cette activité intellectuelle vouée au renouvellement, essentielle pour l'existence de la communauté samburu, se concentre particulièrement au cours des années de l'état de jeunes circoncis. Pendant cette longue période, la mobilité des hommes, due à leur implication dans le soin du bétail et dans la pratique du raid, leur permet d'intégrer plus rapidement que les autres les transformations environnementales et culturelles qui se produisent tant dans leur milieu que dans les territoires les plus lointains. Ce travail d'actualisation des savoirs, de (re)découverte générationnelle et de domestication des espaces est ensuite légué à la réflexion collective par la composition et l'exécution publique, dans des contextes formels, des chants qui racontent leurs voyages et leurs exploits militaires.

Toujours dans The Perception of the Environement, Tim Ingold remet en question la conception « généalogique » de la notion de génération, fondée sur une séparation nette entre individus dans un rapport de descendance. Celle-ci serait à l'origine, selon lui, d'une représentation « hors du temps » et « bidimensionnelle » de l'environnement. Il serait plus opportun de privilégier, dit Ingold, un modèle relationnel qui se manifeste sous forme d'un processus 
vital ininterrompu inscrit dans le territoire comme un « immense agglomérat de sillons entrelacés » (2000, p. 142-151, notre traduction). Le cas des Samburu nous montre que, dans les faits, la séparation sociologique entre générations ${ }^{20}$ ne produit pas forcément la perception d'un territoire inerte sur le plan temporel. Chez les Samburu c'est exactement le contraire qui est vrai. C'est bien par ces coupures générationnelles (Straight, 2005) que la relation avec l'environnement est renouvelée et revitalisée constamment, tout en s'appuyant toujours sur le savoir produit par les promotions précédentes. Chaque génération a pour obligation d'élaborer sa propre réflexion sur le présent et le futur. La modification et la création des répertoires poético-musicaux générationnels sont donc des activités indispensables pour chaque promotion dans l'état de lmurran. Un manque d'engagement dans ce sens serait vu comme un signe de crise inquiétant de toute la société. Le système d'âges apparaît comme un dispositif d'adaptation au changement et d'analyse de l'environnement qui fait de l'instauration de générations successives (et de leur apport intellectuel) son moyen de dominer la contemporanéité. Quand je demandai à un membre du statut des Pères s'il était important pour lui que les membres de la nouvelle promotion perpétuent les chants du passé, il me répondit un peu surpris « Non! Pas du tout ! Les Imurran ne doivent absolument pas répéter nos chants, les chants des vieux. Ils doivent utiliser leurs propres mots! Raconter leurs histoires ${ }^{21} ! \gg$.

\section{Danser autour des chants-arbres}

Les Samburu sont des marcheurs infatigables qui peuvent parcourir des dizaines de kilomètres à pied dans une journée seulement pour conduire leurs bovins au pâturage le plus proche. Les raids, clairement, sont une toute autre histoire. Les villages des populations ennemies se trouvent parfois à plusieurs jours de voyage. Le parcours pour les atteindre représente souvent une véritable épreuve de force et de courage qui se révèle, dans certains cas, plus périlleuse (et meurtrière) que la razzia elle-même. Les Imurran et les jeunes Pères qui les

20. Il faut rappeler que le système des générations samburu auquel on a fait référence ici n'est ni généalogique ni classificatoire. Il est fondé sur un classement des hommes selon leur recrutement dans des promotions générationnelles ouvertes à des intervalles temporels plus ou moins réguliers (Peatrik, 2020). Ceci n'exclue pas la présence d'une conception généalogique des liens de filiation.

21. Conversation avec un ancien de la promotion générationnelle lkishili. Carnet de terrain. LODOKEJEK, 2009. 
accompagnent marchent à grande vitesse en traversant des fleuves, en escaladant des collines parfois pendant trois, cinq jours, en se reposant et en se restaurant le strict nécessaire. Le raid leur demande une dépense d'énergie physique et psychologique immense mais, même en cas de succès, une fois accompli le vol de bétail, leurs peines sont loin d'être terminées. Le chemin du retour, comme l'a montré Serge Tornay chez les Nyangatom d'Éthiopie, peut devenir leur tombe. Les jeunes hommes traversent à rebours le territoire qui les sépare de leurs maisons en courant pendant des jours interminables. Les victimes de leur razzia les traquent. Pas de temps pour s'arrêter ou pour boire une goutte d'eau. Certains meurent de fatigue, d'autres de soif (Tornay, 2001). Quand ils arrivent à destination ils sont au bout de leurs forces. Il n'est donc pas surprenant que leurs chants de raid privilégient autant la description des terres qu'ils ont traversées au détriment de l'action militaire en tant que telle : le parcours du raid peut se transformer en un défi plus redoutable qu'un affrontement aux lances ou aux armes à feu.

Transhumances, nomadisme, déplacements quotidiens vers les pâturages et raids représentent autant d'activités fondatrices du système socio-économique samburu. Toutes partagent un même élément essentiel : la marche vers des territoires plus ou moins éloignés. La capacité à se déplacer sur de longues distances est un attribut indispensable du savoir-faire pastoral des femmes et des hommes adultes ${ }^{22}$. La marche occupe une position capitale dans le mode de vie de cette communauté, au point que même ses danses se présentent sous forme de longs périples à pas rythmés dans la brousse ou en cercle autour d'un groupe de chanteurs. Cette connexion entre mobilité pastorale et pratique chorégraphique est mise en avant par les Samburu eux-mêmes de manière assez explicite : «Danser c'est notre entraînement pour marcher » m'ont-ils dit en plus d'une occasion. Dans une seule session de danse, en effet, certains individus peuvent arriver à effectuer un nombre de pas qui leur permettrait de parcourir jusqu'à dix-douze kilomètres ${ }^{23}$.

Les phrases des solistes, plus que les mélodies vocales, stimulent des réactions kinésiques parfois irrésistibles dans l'auditoire. Le récit chanté du parcours d'un raid peut faire tomber les hommes dans un état second qui les amène à courir sans destination jusqu'à ce qu'un obstacle les arrête. Selon les témoignages

22. Ce n'est pas un hasard si l'un des stéréotypes les plus récurrents chez les Samburu à propos des occidentaux, souvent considérés comme des enfants, est qu'ils « ne savent pas marcher $\gg$.

23. Calcul effectué au moyen d'un podomètre branché à le ceinture d'un danseur. 
que j'ai recueillis chez les jeunes circoncis, en outre, certains mouvements chorégraphiques (comme les sauts) ne seraient qu'une réaction à l'écoute des histoires des razzias qu'eux ou leurs camarades ont accomplies visant à exercer un contrôle sur le corps et éviter de tomber en état second. De toute évidence le lien entre expérience du voyage, relation à l'environnement et danse ne se réduit pas à la seule dimension physique. Marcher pendant des heures en ronde dans la savane ou sauter autour de solistes qui décrivent leurs propres parcours à travers des vallées et des montagnes peuplées d'espèces végétales peut se concevoir comme une modalité de transmission d'une expérience sensorielle, corporelle et visuelle que les hommes et les femmes de la communauté incorporent par la voix des chanteurs. Cette fusion entre parole chantée et mouvement chorégraphique permet, même à ceux qui n'ont pas participé directement aux expéditions des jeunes circoncis, de « pratiquer » (et non seulement de connaître) les espaces et les écosystèmes racontés par les solistes.

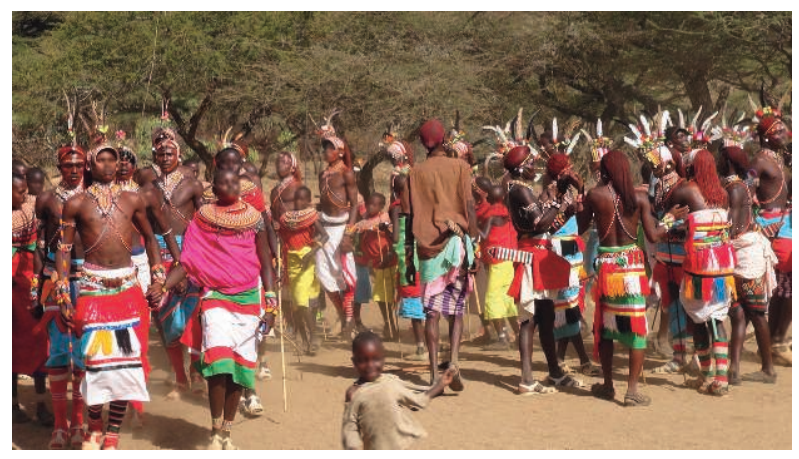

Figure 2

Un groupe de danseurs marche autour d'un chœur (à droite) au sein duquel des solistes, à tour de rôle, décrivent les exploits et les périples des jeunes circoncis de leur unité territoriale.

Phootographie : Giordano Marmone (Simalé, Août 2013)

Le cas de maasani peut éclairer ultérieurement la nature de cette relation. Il s'agit d'une danse qui, dans une large mesure, n'est pas associée à un texte chanté. Son schéma chorégraphique consiste en une longue marche rythmée exécutée par un cortège d'hommes et de femmes qui se tiennent par la main et avancent en colonne par deux ou par trois. Les hommes exécutent des ostinati vocaux alors que le groupe s'éloigne de plus en plus de l'espace de danse originel, tourne autour des arbres, dépasse des obstacles naturels, descend dans des gorges et remonte par des pentes jusqu'à revenir en arrière. Son nom pourrait nous fournir un indice à propos de son origine. La chorégraphie de maasani ressemble en effet 
aux cortèges cérémoniels de la communauté maasai ${ }^{24}$, au cours desquelles des solistes exécutent des textes chantés qui mettent en exergue leur connaissance du territoire et des modalités de mise à mort des lions (Askew \& Odgaard, 2013). Dans le cas de maasani, les danseurs samburu semblent reproduire, sur une échelle réduite, les mouvements, les parcours et les rencontres avec les entités végétales des jeunes circoncis lors de leurs déplacements. L'absence des récits chantés des solistes semble être remplacée par une expérience directe du territoire et de ses composantes. Comme si arbres et chants n'étaient finalement qu'une seule et même chose.

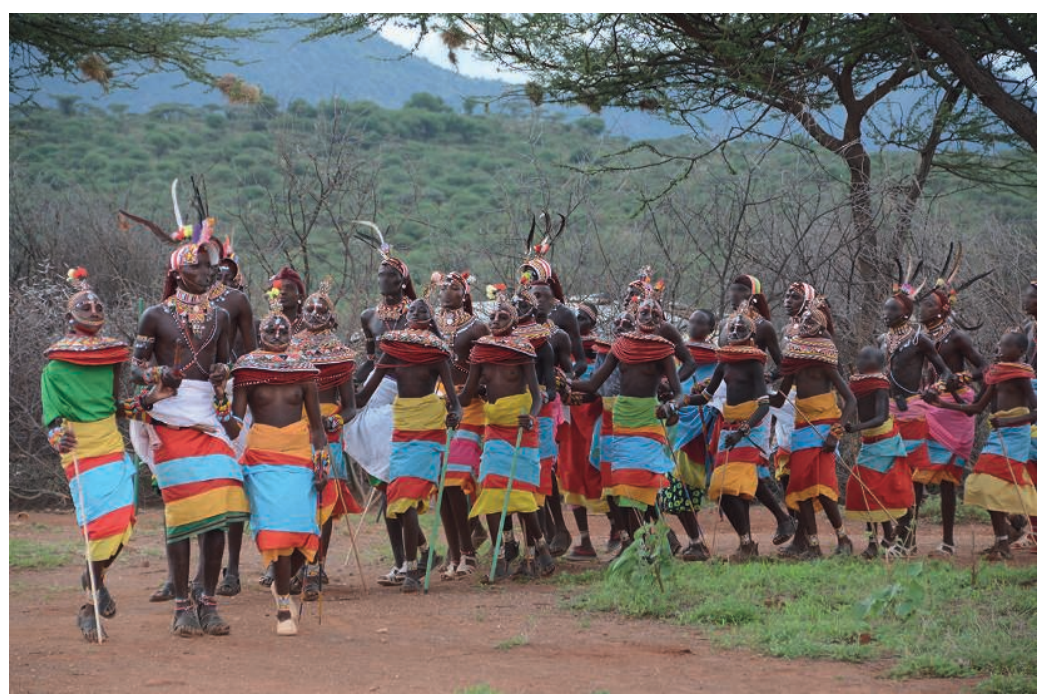

Figure 3

Un groupe de jeunes circoncis et de filles célibataires samburu exécute maasani à l'occasion d'une session de danse cérémonielle.

Photographie : Daniele Sciuto (Lolua, Avril 2012)

\section{Conclusion}

Le raid est un fait social total qui mobilise le système d'âge dans son intégralité et représente une source d'inspiration inépuisable pour la composition de chants et de danses, ce qui fait de lui, indirectement, un instrument d'analyse et de

24. En langue maasai et samburu le terme maasani indique une personne appartenant à la communauté maasai. Le nom de cette danse pourrait aussi dériver de maashani qui peut être traduit par « les errants », PAyne \& Ole-Kotikash, 2008. 
compréhension de la réalité. Les chants composés à l'issue d'une razzia entraînent l'exploration critique d'un macrocosme socio-historique et écologique hétérogène fait de guerre, de politique, d'économie mais aussi, comme nous l'avons vu, de plantes, de fleuves et de montagnes composant un environnement en mutation perpétuelle. Ces éléments du paysage et de l'écosystème constituent le point de jonction entre le proche et le lointain, entre l'ordinaire et l'extra-ordinaire. La perspective que j'ai prise en compte n'est pas seulement celle des individus qui ont été les témoins et les narrateurs de ces événements, mais aussi celle de l'auditoire, composé par des hommes, des femmes et des enfants qui n’ont jamais vu de leurs propres yeux les combats et les lieux décrits par les raiders. Les espèces végétales connues que les lmurran retrouvent dans les territoires de leurs razzias, les fleuves qui s'assèchent aux mêmes rythmes saisonniers dans l' « ailleurs » tout comme dans l'« ici », les toponymes des lieux les plus reculés qui, à travers le contact direct des jeunes circoncis, deviennent familiers, font de la composition des chants de raid un dispositif de découverte, de partage de connaissances mais aussi de domination cognitive sur le « lointain » et sur ce qui est perçu comme « étranger ». La danse joue dans ce contexte un rôle essentiel : les récits chantés de ces longues marches dans des territoires éloignés peuvent, de quelque sorte, être non seulement exécutés et écoutés mais aussi « mis en action » à travers une expérience kinésique coordonnée qui, en transposant la marche elle-même dans le domaine chorégraphique, élargit potentiellement le périmètre de cette relation sensible et cognitive avec les espaces et leurs composantes au-delà du vécu des témoins directs des événements narrés. L'embodied sensation of places, que Steven Feld évoque à propos du chant et de son pouvoir de décrire et exprimer des réalités expérientielles (1996, p. 113), est, dans le cas des Samburu, rendue possible tant par les paroles des solistes que par le mouvement dansé, qui rend le corps un medium de ce processus d'assimilation et de perception de l'environnement.

Le raid, avec les nouvelles relations au territoire qu'il produit et la composition poético-musicale qu'il engendre, est l'espace événementiel, topologique et chronologique au sein duquel la contemporanéité se manifeste en toute sa complexité, à travers les relations politiques, militaires et territoriales que, à chaque fois, il remet en question. À côté de ces plantes familières, de ces recoins de la savane qui deviennent nommables, imaginables et praticables grâce à l'engagement corporel et intellectuel des lmurran et des membres de leur communauté, des technologies exogènes, des nouvelles paroles et des nouveaux réseaux d'interaction socio-économique trouvent leur ancrage dans la réalité quotidienne. C'est ainsi que cette société de pasteurs transhumants maîtrise les changements du monde contemporain, explore des présents alternatifs dans des 
terres au-delà de leurs confins habituels et construit des futurs potentiels percés à jour par des chants, des montagnes et des arbres.

\section{Bibliographie}

Askew Kelly \& OdgaARd Rie, 2013, “The Lions of Lesoit: Shifting Frames og Parakuyo Maasai Indigeneity” in Harris Michelle, Nakata Martin \& CARLson Bronwyn (eds.), The Politics of Identity: Emerging Indigeneity, UTSePress, Sydney, pp. 209-329.

BAsso Keith H., 1988, "Speaking with Names: Language and Landscape among the Western Apache" in Cultural Anthropology, Vol. 3, n² 2, pp. 99-130.

Eczet Jean-Baptiste, 2019, Amour vache. Esthétique sociale en pays mursi (Éthiopie), Éditions Mimésis, Sesto San Giovanni, 370 p.

FELD Steven, 1996, "Waterfalls of Song: An Acoustemology of Place Resounding in Bosavi, Papua New Guinea” in Feld Steven \& BAsso Keith H. (eds.), Senses of Place, School of American Research Press, Santa Fe, pp. 91-135.

Gourlay Kenneth, 1999, “The Making of Karimojong' Cattle Song” in FLOyd Malcolm (ed.), Composing the Music of Africa, Ashgate Publishing Limited, Aldershot, Brookfield, pp. 91-105.

Ingold Tim, 2000, The Perception of the Environment. Essays on livelihood, dwelling and skill, Routledge, London, $465 \mathrm{p}$.

Marmone Giordano, 2020, "The power of youth. Musical practice and the construction of authority among the Samburu (Kenya)" in Ateliers d'Anthropologie, Jeunes en question(s). Onze études de cas en Afrique, $\mathrm{n}^{\circ} 47$, DOI: https://doi.org/10.4000/ateliers.12728.

NANYingi Mark et al., 2008, "Ethnopharmacological survey of Samburu district, Kenya" in Journal of Ethnobiology and Ethnomedicine, $\mathrm{n}^{\circ} 4 / 14$, DOI:10.1186/1746-4269-4-14.

OpIYo Francis et al., 2012, "Resource-based conflicts in drought-prone Northwestern Kenya: The drivers and mitigation mechanisms" in Wudpecker Journal of Agricultural Research, Vol. 1(11), pp. 442-453. 
Payne Doris L., Ole-Kotikash Leonard et al., 2008, Maa Dictionary, University of Oregon, https://darkwing.uoregon.edu/ maasai/Maa\%20 Lexicon/lexicon/main.htm (dictionnaire en ligne).

Peatrik Anne-Marie, 1999, La Vie à pas contés. Génération, Age et Société dans les Hautes Terres du Kenya (Meru Tugania-Igembe), Société d'Ethnologie, Nanterre, 573 p.

Peatrik Anne-Marie, 2013, « Tuer pour engendrer. Les agents d'une masculinité au long cours (Afrique de l'Est) » in Cahiers d'Études Africaines, Vol. 1, n 209-210, p. 217-245.

Peatrik Anne-Marie, 2020, « Pour une anthropologie des jeunes en Afrique » in Ateliers d'anthropologie, $\mathrm{n}^{\circ}$ 47, http://journals.openedition.org/ ateliers/12114; DOI : 10.4000/ateliers.12114.

Spencer Paul, 2004 [1965], The Samburu: A Study of Gerontocracy in a Nomadic Tribe, Routledge and Kegan, London, 376 p.

Spencer Paul, 1985, "Dance as Antithesis in the Samburu Discourse" in Spencer Paul (ed.), Society and the Dance. The Social Anthropology of process and Performance, Cambridge University Press, Cambridge, pp. 140-163.

Straight Bilinda, 2005, "Cutting Time: Beads, Sex and Songs in the Making of Samburu Memory” in James Wendy \& Mills David (eds.), The Qualities of Time: Temporal Dimensions of Social Form and Human Experience, Berg, Oxford and New York, pp. 267-283.

STRAIGHT Bilinda et al., 2016, “'Dust people:' Samburu perspectives on disaster, identity, and landscape" in Journal of Eastern African Studies, Vol. 10, n ${ }^{\circ}$, pp. 168-188.

Tornay, Serge, 2001, Les Fusils jaunes. Génération et Politique en Pays Nyangatom (Ethiopie), Société d'Ethnologie, Nanterre, 363 p.

Résumé : Dans les chants de raid des Samburu, pasteurs transhumants du Kenya, le thème du vol de bétail occupe, curieusement, une place tout à fait marginale. Au lieu de parler de combats, d'ennemis et de vaches volées, les solistes préfèrent se pencher sur le récit des lieux qu'ils ont traversés et de la végétation 
qu'ils ont observée lors de leurs longs périples vers les unités territoriales qu'ils ont l'intention d'attaquer. Selon l'hypothèse que j'explorerai dans cet article, la référence aux plantes, aux collines, aux fleuves et aux autres composants de l'environnement serait fonctionnelle à une domestication des événements et des territoires extra-ordinaires auxquels les jeunes hommes circoncis, avant-garde culturelle de la communauté, font face au cours de leurs expéditions. Le raid apparaît ainsi comme un espace expérientiel qui fournit à la société samburu les moyens matériels et cognitifs pour maîtriser les transformations de la contemporanéité. La danse ainsi que l'exécution et l'écoute de ces chants générationnels inspirés par les parcours des jeunes hommes circoncis au sein d'un environnement changeant permettent aux hommes et aux femmes de cette communauté d'incorporer et de développer des clés de lecture pour interpréter (et donc normaliser) des relations politiques, des systèmes économiques et des espaces de vie qui se modifient constamment.

Mots-clefs : Samburu, environnement, danse, chants, composition, nomadisme, raid, génération, classes d'âge, ethnomusicologie, anthropologie.

Abstract: In the raid songs of the Samburu, transhumant pastoralists of Nothern Kenya, the theme of cattle theft occupies, strangely enough, a marginal place. Instead of talking about fights, enemies and stolen cows, soloists prefer to focus on the story of the places they have crossed and the vegetation they have observed on their long journeys to the territorial units that they intend to attack. According to the bypothesis I will explore in this article, the reference to plants, hills, rivers and other components of the environment are functional to a domestication of extra-ordinary events and territories that circumcised young men, the cultural avant-garde of the community, face during their expeditions. The raid thus appears as an experiential space that provides samburu society with the material and cognitive means to control the transformations of contemporaneity. Dancing, performing and listening these generational songs inspired by the journeys of circumcised young men in a changing environment allow men and women of this community to embody and develop the skills to interpret (and thus normalize) political relations, economic systems and living spaces in constant mutation.

Keywords: Samburu, environment, dance, songs, composition, nomadism, raid, generation, age groups, ethnomusicology, anthropology.

\section{Notes sur l'auteur}

Giordano Marmone est ethnomusicologue et anthropologue. Son travail de recherche porte sur le rôle des pratiques musicales et dansées dans la construction 
CAHIERS DE LITTÉRATURE ORALE

146 Éc(h)opoétiques - $\mathrm{n}^{\circ} 87$

des systèmes sociaux, des temporalités rituelles et des statuts politiques des populations pastorales d'Afrique de l'Est. Il est actuellement postdoctoral fellow de la Fondation Fyssen au Department of Afroamerican and African Studies de l'University of Michigan. 